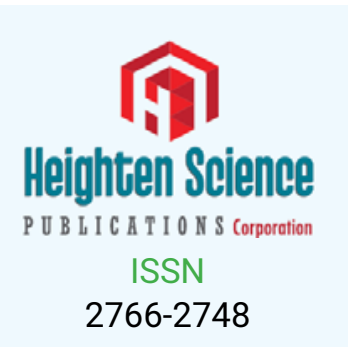

*Address for Correspondence: Zahir Shah Department of Mathematics, Abdul Wali Khan University, Mardan 23200, KP, Pakistan, Email: zahir1987@yahoo.com

Submitted: 27 July 2018

Approved: 16 August 2018

Published: 17 August 2018

Copyright: @2018 Dawar A, et al. This is an open access article distributed under the Creative Commons Attribution License, which permits unrestricted use, distribution, and reproduction in any medium, provided the original work is properly cited

Keywords: SWCNTs; MWCNTs; Nanoparticles; Casson fluids; Thermal radiation; MHD; Parallel Plates; Rotating System; HAM

Check for updates

Review Article

\title{
Magnetohydrodynamic CNTs Casson Nanofluid and Radiative heat transfer in a Rotating Channels
}

\author{
Abdullah Dawar', Zahir Shah²*, Saeed Islam², Muhammad \\ Idress $^{3}$ and Waris Khan ${ }^{3}$ \\ 'Department of Mathematics, Qurtuba University of Science and Information Technology, \\ Peshawar 25000, Pakistan \\ ${ }^{2}$ Department of Mathematics, Abdul Wali Khan University, Mardan 23200, KP, Pakistan \\ ${ }^{3}$ Department of Mathematics, Islamia College University, Mardan 25000, KP, Pakistan
}

\section{Abstract}

The main purpose of this investigation is to inspect the innovative conception of the magneto hydrodynamic (MHD) nanoparticles of single wall carbon nanotubes base on the fluids (water, engine oil, and ethylene, glycol and kerosene oil) between two rotating parallel plates. Carbon nanotubes (CNTs) parade sole assets due to their rare structure. Such structure has significant optical and electronics features, wonderful strength and elasticity, and high thermal and chemical permanence. The heat exchange phenomena is deliberated subject to thermal radiation. Kerosene oil is taken as based nano fluids because of its unique attention due to their advanced thermal conductivities, exclusive features, and applications. The fluid flow is presumed in steady state. With the help of suitable resemblance variables, the fundamental leading equations have been converted to a set of differential equations. To obtain the solution of the modeled problem, the homotopic approach has been used. The influence of imbedded physical variables upon the velocities and temperature profiles are defined and deliberated through graphs. Moreover, for the several values of relevant variables, the skin fraction coefficient and local Nusselt number are tabulated. Plots have been presented in order to examine how the velocities and temperature profile get affected by various flow parameters.

\section{Introduction}

A nanometer-scale tube-like structure is called nanotube. Carbon nanotubes (CNTs) were the first to be discovered in 1952. CNTs are commonly divided into two types called Single-walled carbon nanotubes (SWCNTs) and multi-walled carbon nanotubes (MWCNTs). The potential of CNTs are catalysts, flat panel display, absorption, and shielding of electro-magnetic waves, nanoelectrodes, sensors, supercapicator and energy conversion. Haq et al. [1], Reported that SWCNTs has higher Nusselt number and skin friction than MWCNTs by considering water as a base fluid. Liu et al. [2], Deliberated that ethylene glycol with CNTs have higher thermal conductivities than ethylene glycol suspension without CNTs by studying synthetic engine oil and ethylene glycol in the existence of MWCNTs. The theory of nanofluid past an exponentially stretching sheet has been presented by Nadeem and Lee [3]. Over stretching/shrinking surfaces the mentioned authors [4-7] examined the impacts of nanoparticles for boundary layer flow. Choi [8] was the pioneer who presented the idea of nanofluid by dipping the nanometer-sized particle into the base fluid. For the study of nanofluid flow, the mathematical model was established by Boungiorno [9]. The recent experimental and theoretical study of Sheikholeslami on nanofluids and its applications with different behaviour, properties and effects with uses of different numerical and analytical techniques can be seen in [10-15]. Sheikholeslami and Rokni [16-19] have recently investigated Simulation of nanofluids and $\mathrm{CuOeH} 2 \mathrm{O}$ nanofluid in a curved porous 
enclosure by using mesoscopic approach. Elias et al. [20], have examined the influence of shape on heat transmission. Liquid metals, plasmas, slat water, electrolytes are the examples of such magnetofluids. MHD was presented by Hannes Alfven [21]. Cooling of devices, MHD instruments, crystal growth, electromagnetic casting, and magnetic drug targeting are the real-world uses of MHD in the field of engineering and technologies. Recently Shah et al. [22-25], have studied MHD nanofluid with rotating system.

The determination of current research is to study the nanoparticles of SWCNTs and MWCNTs Casson Fluid based on the fluids (water, engine oil, ethylene glycol and kerosene oil) between two rotating parallel plate under the influence of magnetic parameter and thermal radiation. Kerosene oil is taken as base nanofluid. Casson [26] was the first one who proposed the Casson fluid model, this model characterizes a shear retreating fluid which is presumed to obligate infinite viscosity at zero rate of shear stress. Mehmood et al. $[27,28]$, recently investigated Casson micro polar fluid over a stretching sheet with internal heat transmission by using numerical techniques. Singh Megahe et al. [29], inspected the liquid film flow of Casson fluid in the existence of varied heat flux using slip velocity. Abolbashari et al. [30], studied the Casson nanofluid with entropy generation. The other related study about Casson fluid can be seen in [31-36]. The effect of all embedding parameters has been studied graphically. The analytical result for velocities and temperature profiles are obtained using the HAM technique [37-42].

\section{Some recent application of CNTs}

CNTS used for

- $\quad$ Cancer treatment

- Cardiac autonomic regulation

- In drug delivery

- Platelet activation

- $\quad$ Tissue regeneration

- In solar cell

\section{Mathematical modeling}

Consider the flows of CNTs nanofluid between two parallel plates. The distance between the lower and upper plates is labeled with $h$. SWCNTs and MWCNTs are used as nano-scale materials where kerosene oil is a base liquid. Through thermal radiation, the heat transportation mechanism is examined. Around the $y$-axis the plates are rotated with a constant angular velocity $\gamma$. It should be noted that $\gamma>0$ indicates that both plates rotate in the same direction, $\gamma>0$ indicates that both plates rotate in the opposite directions, $\gamma$ $>0$ is for the static case. The rotation of the lower plate which is moving with velocity $\mathrm{U}$ ${ }_{w=} c x(c>0)$ is quicker than the upper plate. A coordinate system $(x, y, z)$ is chosen in such a way that the $\mathrm{x}$-axis is parallel to the plates, the $\mathrm{y}$-axis is vertical to the plates, and the $\mathrm{z}$-axis is normal to the xy plane. The plates are positioned at $y=0$ and $y=h$. With the help of two forces with same magnitude but opposite direction, the lower plate is being kept stretchable so the position $(0,0,0)$ cannot changes. The fluid flow and heat transfer is supposed in steady state which is incompressible, laminar and stable. Along y direction, the magnetic field $B_{0}$ is substituted with which the fluid is rotating as shown in Figure 1 . The rheological model that illustrates the Casson fluid is known as [34-36]:

$$
\mathrm{T}_{i j}=2\left\{\begin{array}{l}
\left(\mu_{\mathrm{B}}+\frac{\mathrm{P}_{y}}{\sqrt{2 \kappa}}\right) m_{i j}, \quad \kappa>\kappa_{c}, \\
\left(\mu_{\mathrm{B}}+\frac{\mathrm{P}_{y}}{\sqrt{2 \kappa_{c}}}\right) m_{i j}, \quad \kappa>\kappa_{c} .
\end{array}\right.
$$




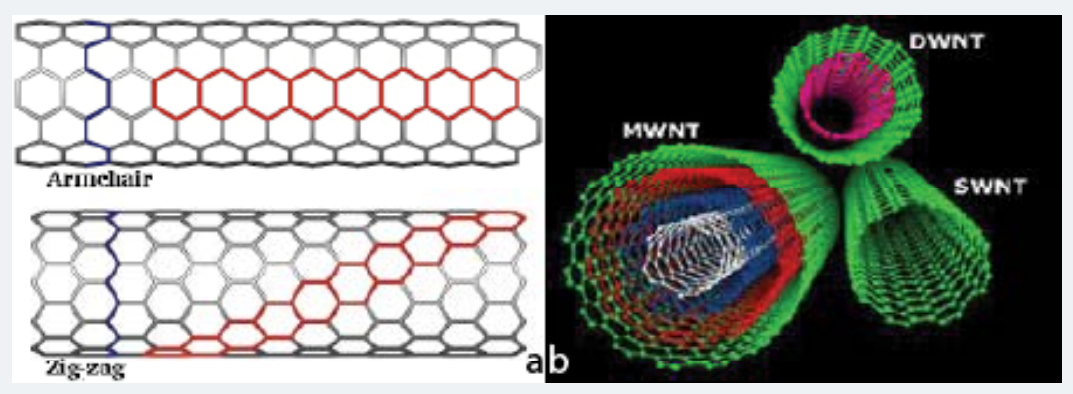

Figure 1: (a). Different types structure of CNTs (b). Structure of SWCNTs and MWCNTs.

$\mathrm{T}_{i j}$ Denotes Cauchy stress tensor, $\mu_{B}$ denotes the dynamic viscosity of the Casson fluid, $k=m_{i j} \cdot m_{i j}$ is the square of components of strain rate $\mathrm{P}_{\mathrm{y}}$ is the yield stress of the fluid and $k_{c}$ represent critical value of $k$

The governing equations for the state of problem are [22-24,37]

$$
\begin{aligned}
& \frac{\partial u}{\partial x}+\frac{\partial u}{\partial y}=0 \\
& \rho_{n f}\left(u \frac{\partial u}{\partial x}+v \frac{\partial u}{\partial y}+2 \gamma w\right)=-\frac{\partial P^{*}}{\partial x}+\left(1+\frac{1}{\beta}\right) \mu_{n f}\left(\frac{\partial^{2} u}{\partial x^{2}}+\frac{\partial^{2} u}{\partial y^{2}}\right)-\sigma_{n f} B_{0}^{2} u, \\
& \rho_{n f}\left(u \frac{\partial v}{\partial x}+v \frac{\partial v}{\partial y}\right)=-\frac{\partial P^{*}}{\partial y}+\mu_{n f}\left(1+\frac{1}{\beta}\right)\left(\frac{\partial^{2} u}{\partial x^{2}}+\frac{\partial^{2} u}{\partial y^{2}}\right), \\
& \rho_{n f}\left(u \frac{\partial w}{\partial x}+v \frac{\partial w}{\partial y}-2 \gamma u\right)=\mu_{n f}\left(1+\frac{1}{\beta}\right)\left(\frac{\partial^{2} w}{\partial x^{2}}+\frac{\partial^{2} w}{\partial y^{2}}\right)+\sigma_{n f} B_{0}^{2} w,
\end{aligned}
$$

Where $P^{*}=P-\frac{\gamma^{2} x^{2}}{2}, \sigma_{n f}, \mu_{n f}$ represent the modified pressure, electrical conductivity, and the dynamic viscosity of nanofluid respectively. In the absence of $P^{*}{ }_{2}$ represents the meshes cross flow beside z-axis and $\beta$ is the parameter of Casson fluid. Mathematically, the phenomenon of heat transmission can be indicated as

$$
u \frac{\partial \mathrm{T}}{\partial x}+v \frac{\partial \mathrm{T}}{\partial y}=\frac{k_{n f}}{(\rho c)_{n f}}\left(\frac{\partial^{2} \mathrm{~T}}{\partial x^{2}}+\frac{\partial^{2} \mathrm{~T}}{\partial y^{2}}\right)-\frac{\partial Q r}{\partial y} .
$$

$\mathrm{T}, \alpha_{n f}=\frac{k_{n f}}{(\rho c)_{n f}}, Q r$ Signifies the fluid temperature, thermal diffusivity and the radiative heat flux respectively. The radiative heat flux is defined as

$$
Q r=-\frac{4 \sigma^{*}}{3 k} \frac{\partial \mathrm{T}^{4}}{\partial y}
$$

Where $\sigma^{*}, k$ indicates the absorption coefficient and Stefan Boltzmann constant respectively.

$$
\begin{array}{r}
\text { As } \mathrm{T}^{4}=4 \mathrm{~T}_{h}^{3} \mathrm{~T}-3 \mathrm{~T}_{h}^{4} \text { the equation (6) becomes } \\
u \frac{\partial \mathrm{T}}{\partial x}+v \frac{\partial \mathrm{T}}{\partial y}=\frac{k_{n f}}{(\rho c)_{n f}}\left(1+\frac{16 \sigma^{*} \mathrm{~T}_{h}^{3}}{3 k}\right) \frac{\partial^{2} \mathrm{~T}}{\partial y^{2}} .
\end{array}
$$


The density, heat capacity, and dynamic viscosity of the nanofluid through mathematical equations are [40].

$$
\begin{gathered}
(\rho c)_{n f}=(1-\varphi) \rho_{f}+\varphi \rho_{C N T}, \quad \rho_{n f}=(1-\varphi)(\rho c)_{f}+\varphi(\rho c)_{C N T}, \\
\mu_{n f}=\frac{\mu_{f}}{(1-\varphi)^{2.5}}, \quad k_{n f}=k_{f}\left(\frac{1-\varphi+2 \varphi \frac{k_{C N T}}{k_{C N T-k_{f}}} \ln \left(\frac{k_{C N T+k_{f}}}{2 k_{f}}\right)}{1-\varphi+2 \varphi \frac{k_{f}}{k_{C N T-k_{f}}} \ln \left(\frac{k_{C N T+k_{f}}}{2 k_{f}}\right)}\right) .
\end{gathered}
$$

Where, $\mu_{f}, \varphi, k_{n f}$ is the dynamic viscosity of base fluid, nanoparticle volumetric friction, and thermal conductivity respectively. The subscripts $C N T, f, n f$ represent carbon nanotubes, base fluid, and nano fluid respectively. $\mathrm{T}_{0}$ is the lower temperature at upper wall and $\mathrm{T}_{h}$ is the higher temperature at lower wall. $\mathrm{T}_{h}$ is retained higher than $\mathrm{T}_{0}$ i.e. $\mathrm{T}_{h}>\mathrm{T}_{0}$. The boundary conditions for the system can be defined as [37].

$$
\begin{aligned}
& \vec{u}=U_{w}=c x, \vec{v}=0, \vec{w}=0, \mathrm{~T}=\mathrm{T}_{h} \text { at } y=0, \\
& \vec{u}=0, \vec{v}=-\mathrm{Z}_{0}, \vec{w}=0, \mathrm{~T}=\mathrm{T}_{0} \text { at } y=h .
\end{aligned}
$$

In equation $(9) \mathrm{Z}_{0}$ is the uniform suction/injection velocity at the upper wall. If $\left(\mathrm{Z}_{0}\right.$ $>0)$ than it is called uniform suction velocity and if $\left(Z_{0}<0\right)$ than it is called uniform injection velocity.

Excluding the pressure gradient and introducing the similarity variables, we have [22-24].

$$
\vec{u}=\operatorname{cxf}^{\prime}(\Gamma), \vec{v}=-\operatorname{chf}(\Gamma), \vec{w}=\operatorname{cxg}(\Gamma), \theta(\Gamma)=\frac{\mathrm{T}-\mathrm{T}_{0}}{\mathrm{~T}_{h}-\mathrm{T}_{0}}, \Gamma=\frac{y}{h} .
$$

The non-dimensional system of equations is

$$
\begin{aligned}
& \left(1+\frac{1}{\beta}\right) f^{i v}(\Gamma)-(1-\varphi)^{2.5}\left[(1-\varphi)+\varphi \frac{\rho_{C N T}}{\rho_{f}}\right] A_{1}\left(f^{\prime}(\Gamma) f^{\prime \prime}(\Gamma)-f(\Gamma) f^{\prime \prime \prime}(\Gamma)\right) \\
& -(1-\varphi)^{2.5}\left[(1-\varphi)+\varphi \frac{\rho_{C N T}}{\rho_{f}}\right] A_{2} g^{\prime}(\Gamma)-\left(\frac{\sigma_{n f}}{\sigma_{f}}\right) \mathrm{M} f^{\prime \prime}(\Gamma)=0, \\
& \left(1+\frac{1}{\beta}\right) g^{\prime \prime}(\Gamma)-(1-\varphi)^{2.5}\left[(1-\varphi)+\varphi \frac{\rho_{C N T}}{\rho_{f}}\right] A_{1}\left(g(\Gamma) f^{\prime}(\Gamma)-f(\Gamma) g^{\prime}(\Gamma)\right) \\
& +2(1-\varphi)^{2.5}\left[(1-\varphi)+\varphi \frac{\rho_{C N T}}{\rho_{f}}\right] A_{2} f^{\prime}(\Gamma)+\left(\frac{\sigma_{n f}}{\sigma_{f}}\right) \operatorname{Mg}(\Gamma)=0, \\
& (1+R d) \theta^{\prime \prime}(\Gamma)+(1-\varphi)^{2.5}\left[(1-\varphi)+\varphi \frac{\rho_{C N T}}{\rho_{f}}\right] \frac{\operatorname{Pr} A_{1}}{\left(\frac{k_{n f}}{k_{f}}\right)} f(\Gamma) \theta^{\prime}(\Gamma)=0,
\end{aligned}
$$

$$
\text { The relevant boundary conditions are } \begin{array}{r}
f(0)=0, f^{\prime}(0)=1, g(0)=0, \theta(0)=1, \\
f(1)=Q, f^{\prime}(1)=0, g(1)=0, \theta(1)=0 .
\end{array}
$$

In equation (14), $Q=Z_{0} / c h$ is the suction and injection parameter. If $(Q>0)$ then it is called suction parameter and if $(Q<0)$ then it is called injection parameter. The above non - dimensional system of equations has the following parameters. 


$$
\begin{aligned}
& A_{1}=\frac{c h^{2}}{v_{f}}(\text { Reynold 's number }), A_{2}=\frac{\gamma h^{2}}{v_{f}}(\text { rotation parameter }), \\
& \operatorname{Pr}=\frac{\mu_{f} c_{p}}{k_{f}}(\text { prandtl number }), R d=\frac{16 \sigma^{*} \mathrm{~T}_{h}^{3}}{3 k k^{*}}(\text { radiation parameter }), \\
& \mathrm{M}=\frac{\sigma_{f} B_{0}^{2} h^{2}}{\rho_{f} v_{f}}(\text { magnetic parameter }), \frac{k_{n f}}{k_{f}}=\frac{k_{s}+2 k_{f}-2 \phi\left(k_{f}-k_{s}\right)}{k_{s}+2 k_{f}+2 \phi\left(k_{f}-k_{s}\right)}, \\
& \frac{\sigma_{n f}}{\sigma_{f}}=1+\frac{3\left(\frac{\sigma_{s}}{\sigma_{f}}-1\right) \phi}{\left(\frac{\sigma_{s}}{\sigma_{f}}+2\right)-\left(\frac{\sigma_{s}}{\sigma_{f}}-1\right) \phi}
\end{aligned}
$$

The skin friction coefficient and local Nusselt number are defined as [38]:

$$
\begin{aligned}
& \tilde{C}_{f}=\frac{\mu_{n f}}{\mu_{f}} f^{\prime \prime}(0) \\
& N u_{x}=-\frac{k_{n f}}{k_{f}}(1+R d) \theta^{\prime}(0) .
\end{aligned}
$$

\section{Solution by HAM}

We use HAM [39-42] to solve Eqs. $(12,13$, and 14) with boundary conditions (15) by the succeeding process.

The primary suppositions are chosen as follows:

$$
\bar{f}(\Gamma)=\Gamma+\frac{1}{2}(Q-1) \Gamma^{2}, \bar{f}_{0}(\Gamma)=0, \bar{\theta}_{0}(\Gamma)=1-\Gamma .
$$

The linear operators are chosen as

$$
\mathrm{L}_{\bar{f}}, \mathrm{~L}_{\bar{g}} \text { and } \mathrm{L}_{\bar{\theta}}: L_{\bar{f}}(\bar{f})=\bar{f}^{\prime \prime}, \mathrm{L}_{\bar{g}}(\overline{\mathrm{g}})=\overline{\mathrm{g}}^{\prime \prime}, \mathrm{L}_{\bar{\theta}}(\bar{\theta})=\bar{\theta}^{\prime \prime} \text {, }
$$

Which have the succeeding properties:

$$
L_{\bar{f}}\left(b_{1}+b_{2} \Gamma+b_{3} \Gamma^{2}\right)=0, \mathrm{~L}_{\bar{g}}\left(b_{4}+b_{5} \Gamma\right)=0, \mathrm{~L}_{\bar{\theta}}\left(b_{6}+b_{7} \Gamma\right)=0 \text {, }
$$

Where $b_{i}(i=1-7)$.

The consequence non-linear operators $N_{\bar{f}}, N_{\bar{g}}$ and $N_{\bar{\theta}}$ are indicated as:

$$
\begin{aligned}
& N_{\bar{f}}[\bar{f}(\Gamma ; \psi), \bar{g}(\Gamma ; \psi)]=\left(1+\frac{1}{\beta}\right) \frac{\partial^{4} \bar{f}(\Gamma ; \psi)}{\partial \Gamma^{4}} \\
& -(1-\varphi)^{2.5}\left[(1-\varphi)+\varphi \frac{\rho_{C N T}}{\rho_{f}}\right] A_{1}\left\{\begin{array}{l}
\frac{\partial \bar{f}(\Gamma ; \psi)}{\partial \Gamma} \frac{\partial^{2} \bar{f}(\Gamma ; \psi)}{\partial \Gamma^{2}}- \\
\bar{f}(\Gamma ; \psi) \frac{\partial^{3} \bar{f}(\Gamma ; \psi)}{\partial \Gamma^{3}}
\end{array}\right\} \\
& -(1-\varphi)^{2.5}\left[(1-\varphi)+\varphi \frac{\rho_{C N T}}{\rho_{f}}\right] A_{2} \frac{\partial \bar{g}(\Gamma ; \psi)}{\partial \Gamma}-\left(\frac{\sigma_{n f}}{\sigma_{f}}\right) M \frac{\partial^{2} \bar{f}(\Gamma ; \psi)}{\partial \Gamma^{2}},
\end{aligned}
$$




$$
\begin{aligned}
& N_{\bar{g}}[\bar{f}(\Gamma ; \psi), \bar{g}(\Gamma ; \psi)]=\left(1+\frac{1}{\beta}\right) \frac{\partial^{2} \bar{g}(\Gamma ; \psi)}{\partial \Gamma^{2}} \\
& -(1-\varphi)^{2.5}\left[(1-\varphi)+\varphi \frac{\rho_{C N T}}{\rho_{f}}\right] A_{1}\left(\begin{array}{l}
\bar{g}(\Gamma ; \psi) \frac{\partial \bar{f}(\Gamma ; \psi)}{\partial \Gamma} \\
\left.-\bar{f}(\Gamma ; \psi) \frac{\partial \bar{g}(\Gamma ; \psi)}{\partial \Gamma}\right)
\end{array}\right) \\
& +2(1-\varphi)^{2.5}\left[(1-\varphi)+\varphi \frac{\rho_{C N T}}{\rho_{f}}\right] A_{2} \frac{\partial \bar{f}(\Gamma ; \psi)}{\partial \Gamma}+\left(\frac{\sigma_{n f}}{\sigma_{f}}\right) M \bar{g}(\Gamma ; \psi), \\
& N_{\bar{\theta}}[\bar{f}(\Gamma ; \psi), \bar{\theta}(\Gamma ; \psi)]=(1+R d) \frac{\partial^{2} \bar{\theta}(\Gamma ; \psi)}{\partial \Gamma^{2}}+\left[(1-\varphi)+\varphi \frac{\left(\rho c_{p}\right)_{C N T}}{\left(\rho c_{p}\right)_{f}}\right] \frac{\operatorname{Pr} A_{1}}{\left(\frac{k_{n f}}{k_{f}}\right)} \bar{f}(\Gamma, \psi) \frac{\partial \bar{\theta}(\Gamma ; \psi)}{\partial \Gamma} .
\end{aligned}
$$

The zero $^{\text {th }}$ order problems from In organize to solve Eqs. $(12,13$, and 14) are:

$$
\begin{aligned}
& (1-\psi) L_{\bar{f}}\left[\bar{f}(\Gamma ; \psi)-\bar{f}_{0}(\Gamma)\right]=\psi \hbar_{\bar{f}} N_{\bar{f}}[\bar{f}(\Gamma ; \psi), \bar{g}(\Gamma ; \psi)], \\
& (1-\psi) L_{\bar{g}}\left[\bar{g}(\Gamma ; \psi)-\bar{g}_{0}(\Gamma)\right]=\psi \hbar_{\bar{g}} N_{\bar{g}}[\bar{f}(\Gamma ; \psi), \bar{g}(\Gamma ; \psi)], \\
& (1-\psi) L_{\bar{\theta}}\left[\bar{\theta}(\Gamma ; \psi)-\bar{\theta}_{0}(\Gamma)\right]=\psi \hbar_{\bar{\theta}} N_{\bar{\theta}}[\bar{f}(\Gamma ; \psi), \bar{\theta}(\Gamma ; \psi)] .
\end{aligned}
$$

The equivalent boundary conditions are:

$$
\begin{aligned}
& \left.\bar{f}(\Gamma ; \psi)\right|_{\Gamma=0}=0,\left.\frac{\partial \bar{f}(\Gamma ; \psi)}{\partial \Gamma}\right|_{\Gamma=0}=1,\left.\bar{f}(\Gamma ; \psi)\right|_{\Gamma=1}=Q,\left.\frac{\partial \bar{f}(\Gamma ; \psi)}{\partial \Gamma}\right|_{\Gamma=1}=0, \\
& \left.\bar{g}(\Gamma ; \psi)\right|_{\Gamma=0}=0,\left.\bar{g}(\Gamma ; \psi)\right|_{\Gamma=1}=0, \\
& \left.\bar{\theta}(\Gamma ; \psi)\right|_{\Gamma=0}=1,\left.\bar{\theta}(\Gamma ; \psi)\right|_{\Gamma=1}=0,
\end{aligned}
$$

Where $\psi \in[0,1]$ is the emerging parameter? In case of $\psi=0$ and $\psi=1$ we have:

$$
\bar{f}(\Gamma ; 1)=\bar{f}(\Gamma), \bar{g}(\Gamma ; 1)=\bar{g}(\Gamma) \text { and } \bar{\theta}(\Gamma ; 1)=\bar{\theta}(\Gamma) \text {, }
$$

Expanding $\bar{f}(\Gamma ; \psi), \bar{g}(\Gamma ; \psi)$ and $\bar{\theta}(\Gamma ; \psi)$ by Taylor's series

$$
\begin{aligned}
& \bar{f}(\Gamma ; \psi)=\bar{f}_{0}(\Gamma)+\sum_{q=1}^{\infty} \bar{f}_{q}(\Gamma) \psi^{q}, \\
& \bar{g}(\Gamma ; \psi)=\bar{g}_{0}(\Gamma)+\sum_{q=1}^{\infty} \bar{g}_{q}(\Gamma) \psi^{q}, \\
& \bar{\theta}(\Gamma ; \psi)=\bar{\theta}_{0}(\Gamma)+\sum_{q=1}^{\infty} \bar{\theta}_{q}(\Gamma) \psi^{q} .
\end{aligned}
$$

\section{Where}

$$
\bar{f}_{q}(\Gamma)=\left.\frac{1}{q !} \frac{\partial \bar{f}(\Gamma ; \psi)}{\partial \Gamma}\right|_{\psi=0}, \overline{\mathrm{g}}_{q}(\Gamma)=\left.\frac{1}{q !} \frac{\partial \bar{g}(\Gamma ; \psi)}{\partial \Gamma}\right|_{\psi=0} \text { and } \bar{\theta}_{q}(\Gamma)=\left.\frac{1}{q !} \frac{\partial \bar{\theta}(\Gamma ; \psi)}{\partial \Gamma}\right|_{\psi=0} \text {. }
$$

As the series (31) converges at $\psi=1$, changing $\psi=1$ in (31), we get: 


$$
\begin{aligned}
& \bar{f}(\Gamma)=\bar{f}_{0}(\Gamma)+\sum_{q=1}^{\infty} \bar{f}_{q}(\Gamma), \\
& \bar{g}(\Gamma)=\bar{g}_{0}(\Gamma)+\sum_{q=1}^{\infty} \bar{g}_{q}(\Gamma), \\
& \bar{\theta}(\Gamma)=\bar{\theta}_{0}(\Gamma)+\sum_{q=1}^{\infty} \bar{\theta}_{q}(\Gamma) .
\end{aligned}
$$

The $q^{\text {th }}$-order problem gratifies the following:

$$
\begin{aligned}
& L_{\bar{f}}\left[\bar{f}_{q}(\Gamma)-\chi_{q} \bar{f}_{q-1}(\Gamma)\right]=\hbar_{\bar{f}} U_{q}^{\bar{f}}(\Gamma), \\
& L_{\bar{g}}\left[\bar{g}_{q}(\Gamma)-\chi_{q} \bar{g}_{q-1}(\Gamma)\right]=\hbar_{\bar{g}} U_{q}^{\bar{g}}(\Gamma), \\
& L_{\bar{\theta}}\left[\bar{\theta}_{q}(\Gamma)-\chi_{q} \bar{\theta}_{q-1}(\Gamma)\right]=\hbar_{\bar{\theta}} U_{q}^{\bar{\theta}}(\Gamma) .
\end{aligned}
$$

The equivalent boundary conditions are:

$$
\begin{aligned}
& \bar{f}_{q}(0)=\bar{f}_{q}^{\prime}(0)=\bar{f}_{q}(1)=\bar{f}_{q}^{\prime}(1)=0, \\
& \bar{g}_{q}(0)=\bar{g}_{q}(1)=0, \\
& \bar{\theta}_{q}(0)=\bar{\theta}_{q}(1)=0 .
\end{aligned}
$$

Here

$$
\begin{aligned}
& U_{q}^{\bar{f}}(\Gamma)=\left(1+\frac{1}{\beta}\right) \bar{f}_{q-1}^{i v}-A_{1}(1-\varphi)^{2.5}\left[(1-\varphi)+\varphi \frac{\rho_{C N T}}{\rho_{f}}\right]\left(\sum_{k=0}^{q-1} \bar{f}_{q-1-k}^{\prime} \bar{f}_{k}^{\prime \prime}-\sum_{k=0}^{q-1} \bar{f}_{q-1-k} \bar{f}_{k}^{\prime \prime \prime}\right) \\
& -A_{2}(1-\varphi)^{2.5}\left[(1-\varphi)+\varphi \frac{\rho_{C N T}}{\rho_{f}}\right] \bar{g}_{q-1}-\left(\frac{\sigma_{n f}}{\sigma_{f}}\right) M \bar{f}_{q-1}^{\prime \prime}, \\
& U_{q}^{\bar{g}}(\Gamma)=\left(1+\frac{1}{\beta}\right) \bar{g}_{q-1}^{\prime \prime}-(1-\varphi)^{2.5}\left[(1-\varphi)+\varphi \frac{\rho_{C N T}}{\rho_{f}}\right] A_{1}\left(\sum_{k=0}^{q-1} \bar{g}_{q-1-k} \bar{f}_{k}^{\prime}-\sum_{k=0}^{q-1} \bar{f}_{q-1-k} \bar{g}_{k}^{\prime}\right) \\
& +2(1-\varphi)^{2.5}\left[(1-\varphi)+\varphi \frac{\rho_{C N T}}{\rho_{f}}\right] A_{2} \bar{f}_{q-1}^{\prime}+\left(\frac{\sigma_{n f}}{\sigma_{f}}\right) M \bar{g}_{q-1}, \\
& U_{q}^{\bar{\theta}}(\Gamma)=(1+R d) \bar{\theta}_{q-1}^{\prime \prime}+\left[(1-\varphi)+\varphi \frac{\left(\rho c_{p}\right)_{C N T}}{\left(\rho c_{p}\right)_{f}}\right] \frac{\operatorname{Pr} A_{1}}{\left(\frac{k_{n f}}{k_{f}} \sum_{k=0}^{q-1} \bar{f}_{q-1-k} \bar{\theta}_{k}^{\prime \prime} .\right.}
\end{aligned}
$$

Where

$$
\chi_{q}= \begin{cases}0, & \text { if } \psi \leq 1 \\ 1, & \text { if } \psi>1\end{cases}
$$

\section{Results and Discussion}

In order to investigate the flow and heat transfer performance for both SWCNTs and MWCNTs based on kerosene nanoliquids between two rotating parallel plate, (Figure 3-16) are plotted. Figure 1 displays different structure of CNTs and Figure 2 shows physical shape of the flow. Figures 3-7 are plotted to see the impact of various parameters on velocity and temperature profiles for both SWCNTs and MWCNTs- 

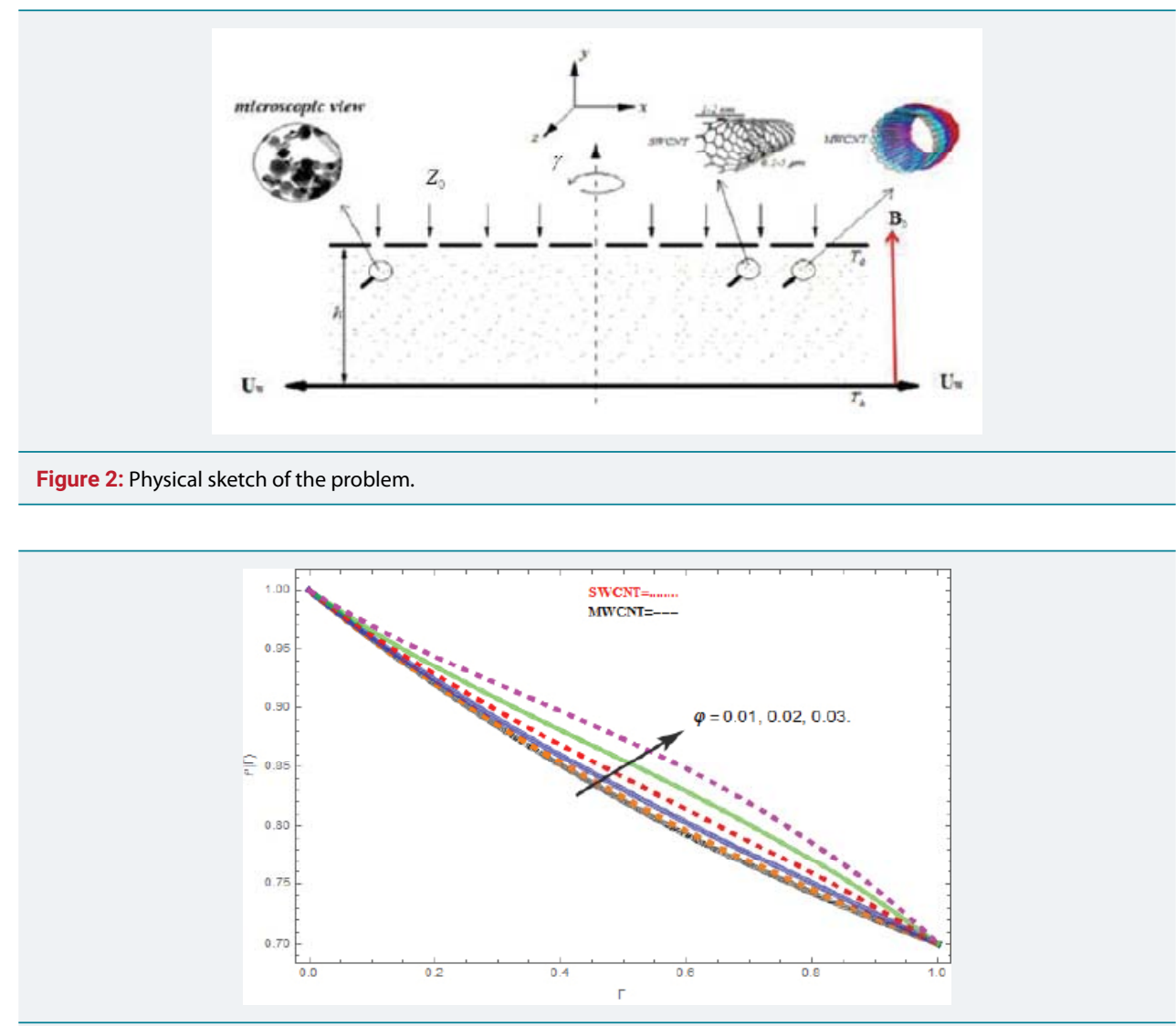

Figure 3: The outcome of $\otimes$ on $f^{\prime}(\Gamma)$, when $A_{1}=0.5, A_{2}=0.6, Q=0.8, M=1.0$.

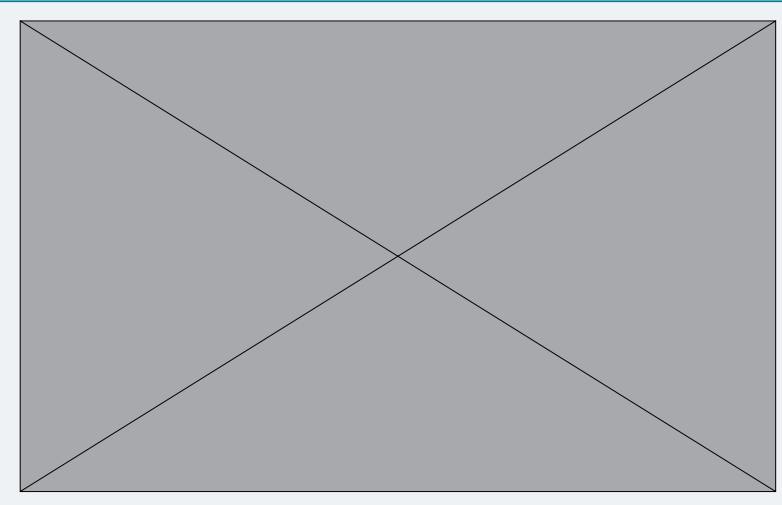

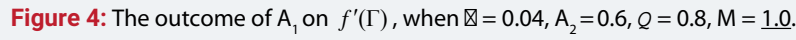

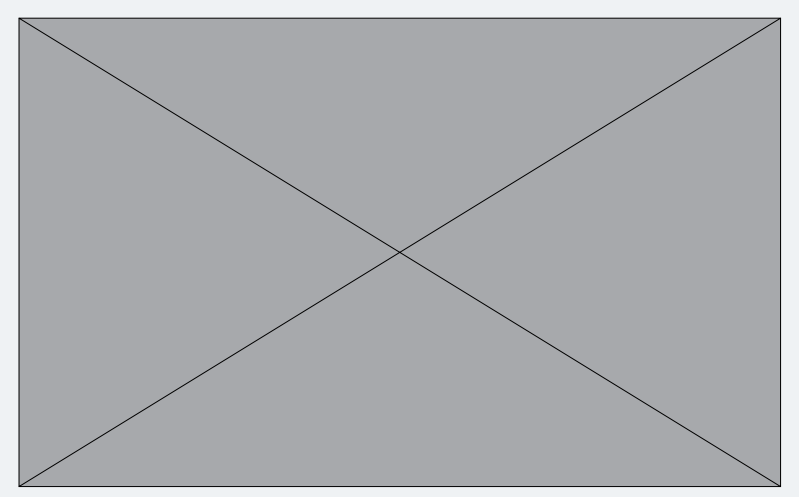

Figure 5: The outcome of $A_{2}$ on $f^{\prime}(\Gamma)$, when $\mathbb{\nabla}=0.04, A_{1}=0.5, Q=0.8, M=\underline{1.0}$. 


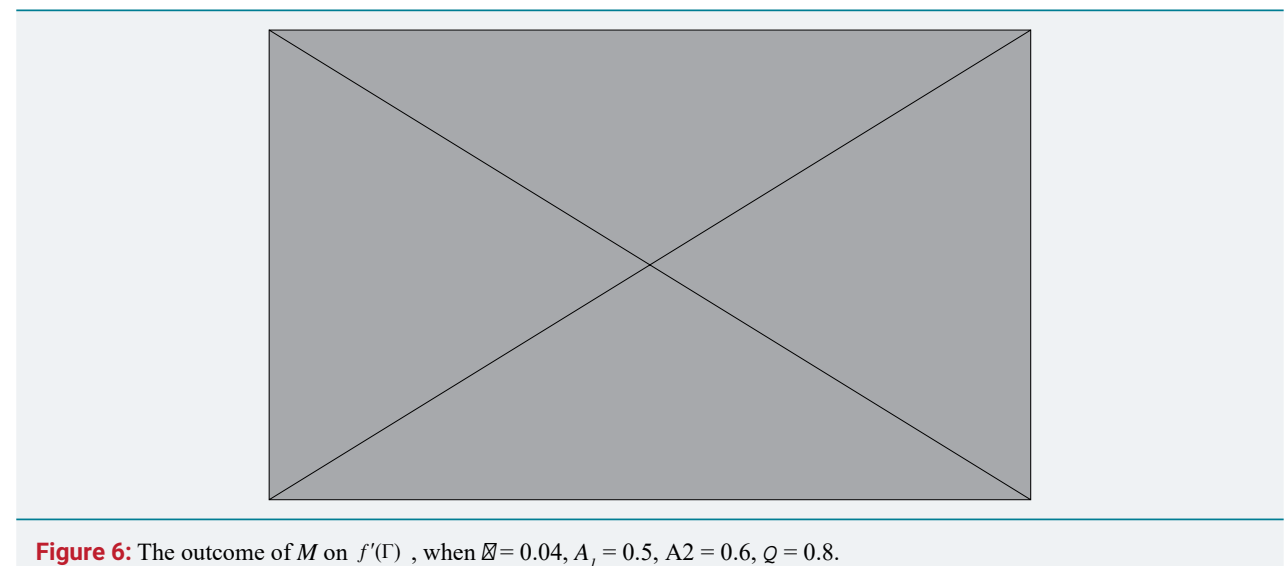

Figure 6: The outcome of $M$ on $f^{\prime}(\Gamma)$, when $\otimes=0.04, A_{1}=0.5, \mathrm{~A} 2=0.6, Q=0.8$.

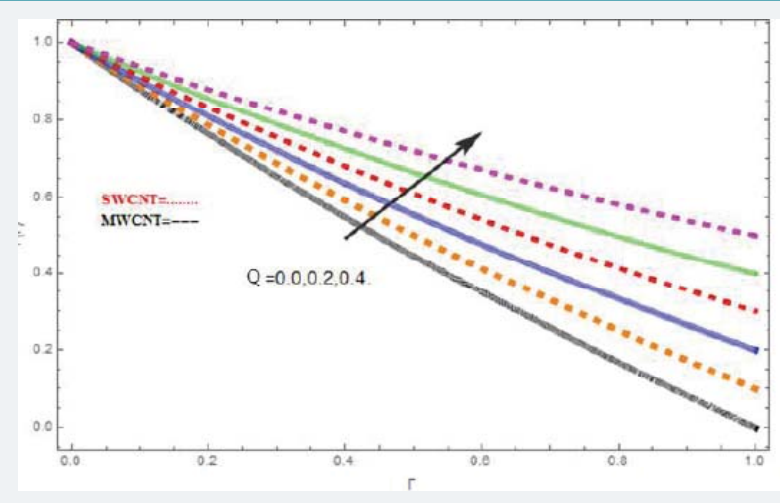

Figure 7: The outcome of suction $(Q<0)$ on $f^{\prime}(\Gamma)$, when $\otimes=0.04, A_{1}=0.5, A_{2}=0.6, M=1.0$.

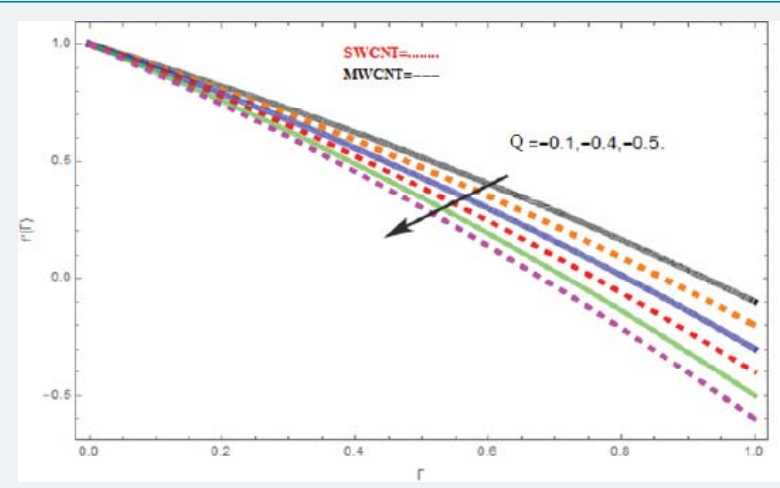

Figure 8: The outcome of injection $(Q<0)$ on $f^{\prime}(\Gamma)$, when $\otimes=0.04, A_{1}=0.5, A_{2}=0.6, M=1.0$.

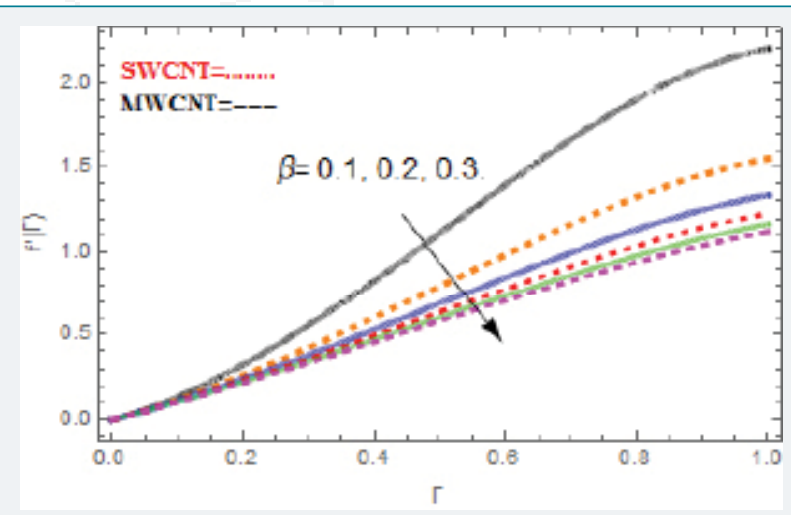

Figure 9: The outcome of $\beta$ on $f^{\prime}(\Gamma)$, when $\otimes=0.04, A_{1}=0.5, A_{2}=0.6, M=1.0$. 


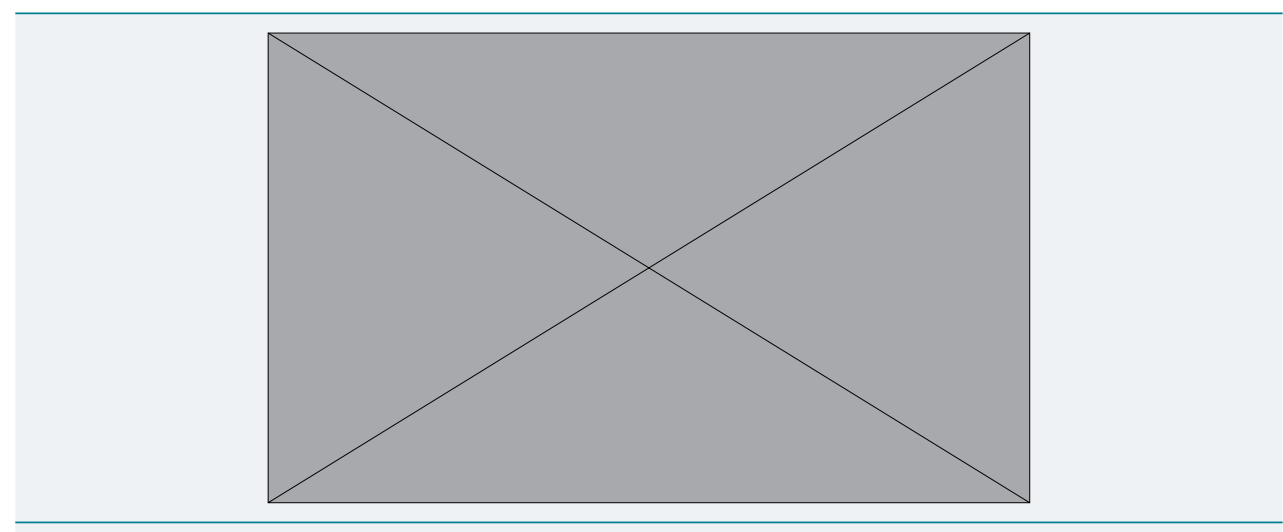

Figure 10: The outcome of $\forall$ on $g(\Gamma)$, when $\mathrm{A}_{1}=0.5, \mathrm{~A}_{2}=0.6, \mathrm{M}=1.0$.

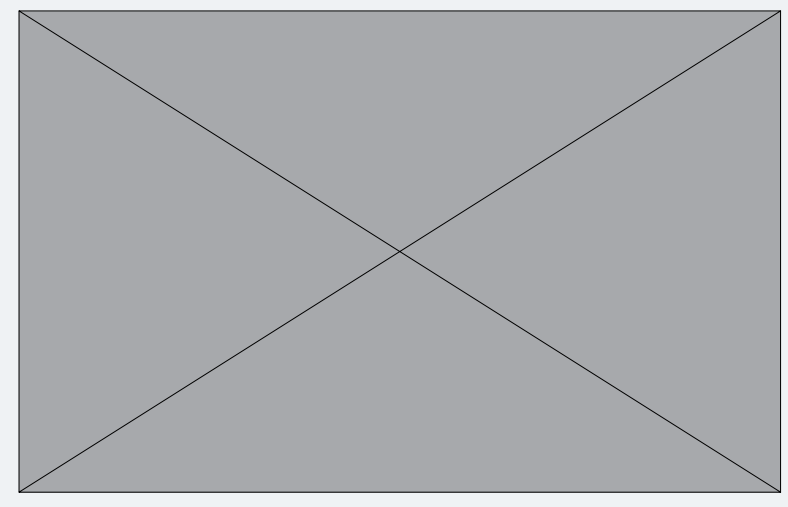

Figure 11: The outcome of $A_{1}$ on $g(\Gamma)$, when $\nabla=0.04, A_{2}=0.6, M=1.0$.

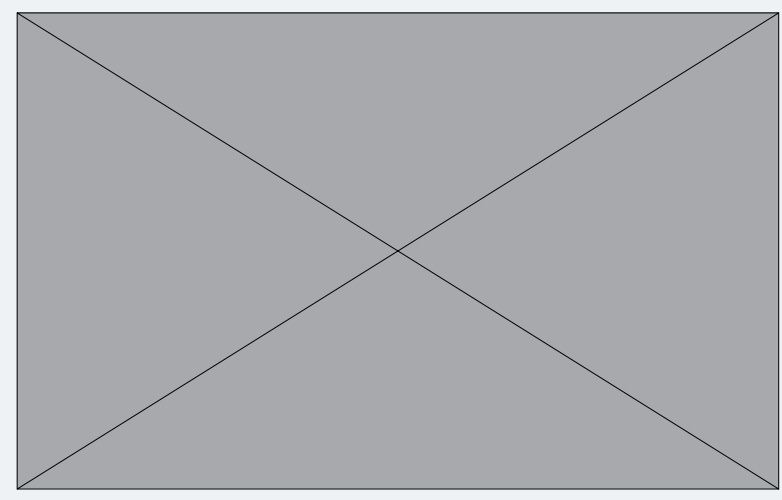

Figure 12: The outcome of $\mathrm{A}_{2}$ on $g(\Gamma)$, when $\otimes=0.04, \mathrm{~A}_{1}=0.5, \mathrm{M}=1.0$.

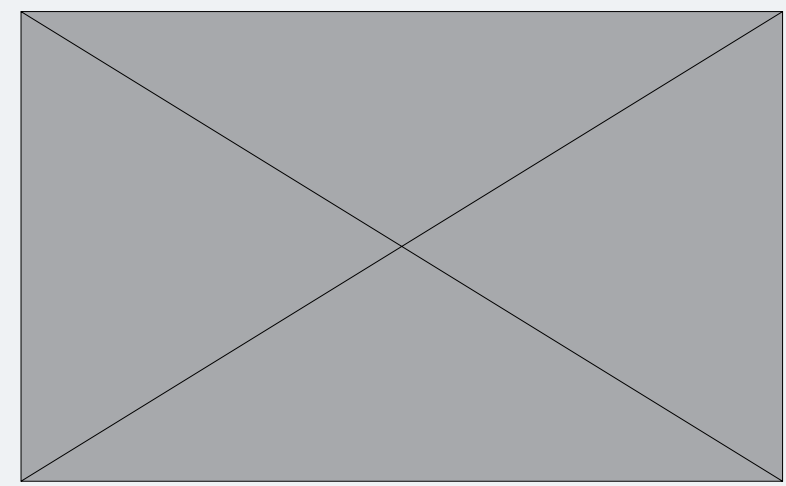

Figure 13: The outcome of $\mathrm{M}$ on $g(\Gamma)$, when $\otimes=0.04, \mathrm{~A}_{1}=0.5, \mathrm{~A}_{2}=06$.

Published: August 17, 2018 


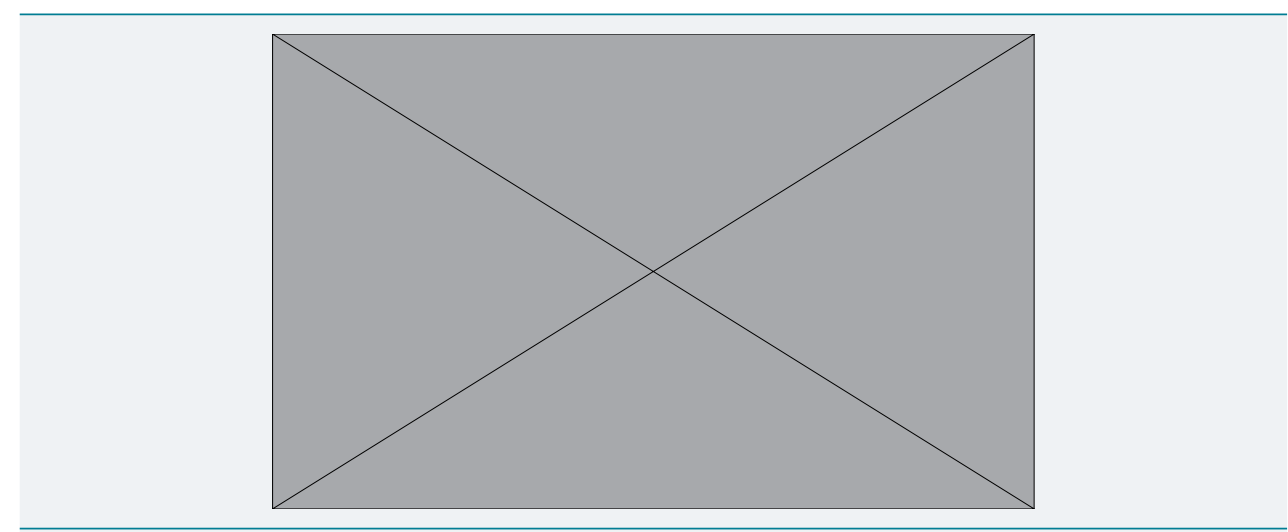

Figure 14: The outcome of $\otimes$ on $\theta(\Gamma)$, when $\mathrm{A}_{1}=0.5, \operatorname{Pr}=7.1, \mathrm{Rd}=0.9$.

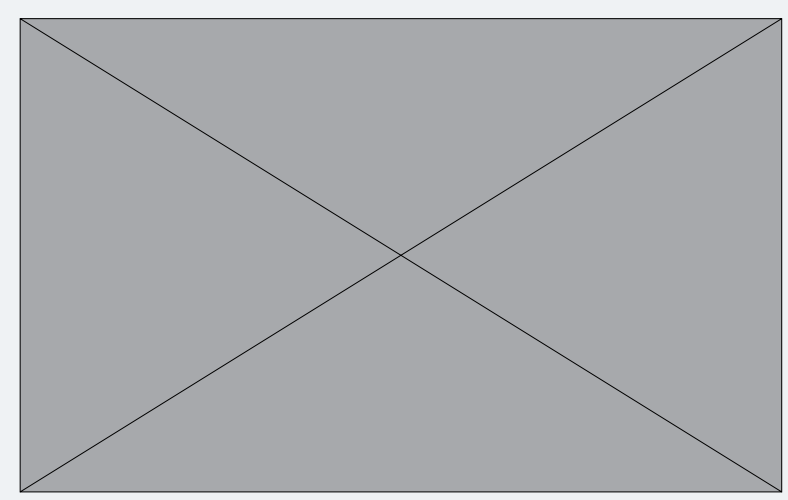

Figure 15: The outcome of $A_{1}$ on $\theta(\Gamma)$, when $\otimes=0.04, \operatorname{Pr}=7.1, \operatorname{Rd}=0.9 . M=1.0$.

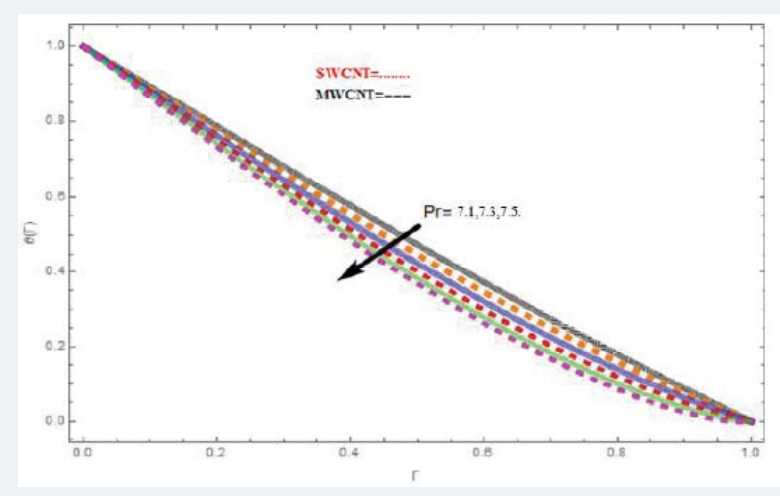

Figure 16: The outcome of $\operatorname{Pr}$ on $\theta(\Gamma)$, when $\otimes=0.04, \mathrm{~A}_{1}=0.5, \operatorname{Pr}=0.9$.

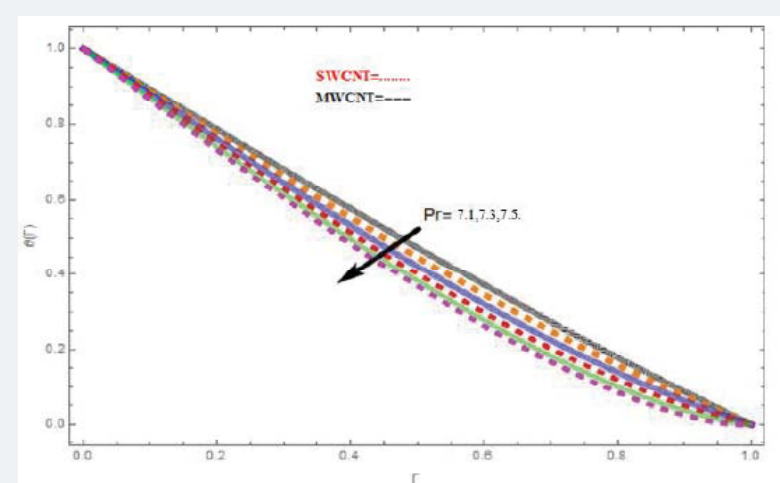

Figure 17: The outcome of $\operatorname{Rd}$ on $\theta(\Gamma)$, when $\otimes=0.04, \mathrm{~A}_{1}=0.5, \operatorname{Pr}=0.9$ 
kerosene nanoliquids. These parameters are nanoparticles volume friction $(\bigotimes)$, Reynolds number $\left(A_{1}\right)$, Rotation parameter $\left(\mathrm{A}_{2}\right)$, Magnetic parameter $(\mathrm{M})$, Suction parameter $(Q>0)$ and Injection parameter $(Q<0)$ respectively. Figure 3 is plotted to see the comparison between the SWCNTs and MWCNTs with the escalating values of nanoparticle volume fraction $(\bigotimes)$. From Figure 3 we observed that there is petty disparity in $f^{\prime}(\Gamma)$ with the escalating values of $(\bigotimes)$. We also observed that the MWCNTs has comparatively much greater $f^{\prime}(\Gamma)$ as compared to SWCNTs. Figure 4 is plotted to see the impact of $\left(A_{1}\right)$ on $f^{\prime}(\Gamma)$ with the different escalating values. We observed from the figure, that with the different escalating values of $\left(A_{1}\right)$, the velocity profile $f^{\prime}(\Gamma)$ changes its behavior from rising to reducing at the medium of the two plates (i.e. $\Gamma=0.5)$ . This is because of stretching of the lower plates. Figure 5 is plotted to see the impact of $\left(A_{2}\right)$ on $f^{\prime}(\Gamma)$. From figure 5 , we observed that the disparity in $f^{\prime}(\Gamma)$ gives dual behavior. Within the region $(0 \leq \Gamma \leq 0.5)$, due to numerous escalating values of $\left(A_{2}\right)$ the velocity profile $f^{\prime}(\Gamma)$ shows declining behavior, however within the region $(0.5<\Gamma \leq 1), f^{\prime}(\Gamma)$ gives escalating behavior for various escalating values of $\left(A_{2}\right)$. In addition, at the upper half of the channel $\left(A_{2}\right)$ gives more dominant variation for $f^{\prime}(\Gamma)$. Figure 6 is designed to see the influence of $(\mathrm{M})$ on $f^{\prime}(\Gamma)$. According to Lorentz force theory the magnetic field parameter has reverse effect on $f^{\prime}(\Gamma)$, that is $f^{\prime}(\Gamma)$ reduces with the escalation in $(M)$. Figure 7 and 8 are plotted to see the impact of suction $(Q>0)$ and injection $(Q<$ 0 ) parameters at the upper plate for $f^{\prime}(\Gamma)$. Form figure 7 , we see that $f^{\prime}(\Gamma)$ escalates with suction $(Q<0)$, that is $f^{\prime}(\Gamma)$ increases with positive the values of $(Q)$, while form Figure 8, it is observed that $f^{\prime}(\Gamma)$ reduces with injection $(Q<0)$, that is $f^{\prime}(\Gamma)$ reduces with the negative $(Q)$. It is fairly obvious that the presence of CNTs nanoparticles has improved the velocity function $f^{\prime}(\Gamma)$, while the velocity function $f^{\prime}(\Gamma)$ increases more when $(Q>0)$ is present. But the decrement in velocity function $f^{\prime}(\Gamma)$ is due to the fact that $(Q<0)$ absorbs the internal heat energy from the surface. Figure 9 is plotted to see the impact of $(\beta)$ on $f^{\prime}(\Gamma)$. From here we observed that the increasing values of $(\beta)$ shows reduction in $f^{\prime}(\Gamma)$. Figure 10 is plotted to see the impact of $(\bigotimes)$ on $g(\Gamma)$.

From Figure 10 we observed that there is petty disparity in $g(\Gamma)$ with the escalating values of $(\bigotimes)$. We also observed that the MWCNTs has comparatively much greater $g(\Gamma)$ as compared to SWCNTs. Figure 11 is plotted to see the impact of on $\left(A_{1}\right)$ $g(\Gamma)$. Figure 11 shows reduction in $g(\Gamma)$ between the two rotating plates with the increasing values of $\left(A_{1}\right)$ and the position of the maximum amount of $g(\Gamma)$ approaches to the stretching sheet. Figure 12 is plotted to see the impact of $\left(A_{2}\right)$ on $g(\Gamma)$. From Figure 12, we observed that the increasing values of $\left(A_{2}\right)$ gives decreasing behavior to $g(\Gamma)$. It is also observed that the disturbance in $g(\Gamma)$ is higher at the medium of the channels as compared to upper and lower surface of the channels. Figure 13 is plotted to see the impact of $(M)$ on $g(\Gamma)$. According to Lorentz force theory the magnetic field parameter has reverse effect on $g(\Gamma)$, that is $g(\Gamma)$ reduces with the escalation in $(M)$. Figure 14 is plotted to see the comparison between the SWCNTs and MWCNTs with the escalating values of nanoparticle volume fraction $(\bigotimes)$. From figure 14 we observed that there is petty disparity in $\theta(\Gamma)$ with the escalating values of $(\bigotimes)$.

We also observed that the MWCNTs has comparatively much greater $\theta(\Gamma)$ as compared to SWCNTs. Figure 15 is plotted to see the impact of $\left(A_{1}\right)$ on $\theta(\Gamma)$. From here we observed that the escalating value of $\left(A_{1}\right)$ shows escalation in $\theta(\Gamma)$. As the distance from the surface escalates, $\theta(\Gamma)$ decreases. Figure 16 is plotted to see the impact of $(\mathrm{Pr})$ on $\theta(\Gamma)$. From here we see that the escalating values of $(\operatorname{Pr})$ shows reduction in $\theta(\Gamma)$ . Physically, the nanofluids have a large thermal diffusivity with small $(P r)$, but this effect is revers for higher $(\mathrm{Pr})$, therefore the temperature of liquid shows decreasing behavior. Figure 17 is plotted to see the impact of $(R d)$ on $\theta(\Gamma)$. Thermal radiation has leading rule in heat transmission when the coefficient of convection heat transmission is small. From here we see that the escalating values of $(R d)$ shows acceleration in $\theta(\Gamma)$. 


\section{Discussion}

Tables 1,2 are schemed to see the influences of different embedding parameters on skin fraction coefficient $f^{\prime}(\Gamma)$ and local Nusselt number $\left(N u_{x}\right)$. From Table 1, we see that the effect of $A_{1}$ on skin fraction coefficient. It is clear from the table that at $f^{\prime}(\Gamma)$, the table shows increasing behavior to 0.3 but from 0.3 to 0.5 , the table values show decreasing behavior. At the medium of the channel the skin friction coefficient changes its behavior from increasing to decreasing. This is because of stretching of the lower plates. It is also clear form the table that the escalating values of $A_{2}$ shows reducing behavior. The escalating values of $(M)$ shows decreasing behavior in skin fraction coefficient $f^{\prime}(\Gamma)$. It is due to Lorentz force theory. The increasing and decreasing values of suction/injection parameter $(Q)$ show two different behaviors. The escalating values of injection parameter $(Q<0)$ show decreasing behavior and also the escalating values of suction parameter $(Q>0)$ show increasing behavior in skin fraction $f^{\prime}(\Gamma)$ . From Table 2, we see that the increasing values of $\left(A_{1}\right)$ and positive $Q$ (i.e. $Q>0$ ) show increasing behavior in local Nusselt number $\left(N u_{x}\right)$, while $\left(A_{2}\right),(\mathrm{M})$ and negative $Q$ (i.e. $Q>0$ ) show decreasing behavior in local Nusselt number $\left(N u_{x}\right)$. Tables 3-5 are schemed to study the Physical properties of CNTs, thermo physical properties CNTs and nanofluids of some base fluids, Thermal conductivity $\left(k_{n f}\right)$ of CNTs with different volume fraction $(\bowtie)$ respectively.

\section{Conclusion}

The investigation for the two-dimensional flow of kerosene oil based nanofluid over an inclined stretching sheet with suction/injection, MHD and radiative heat flux effects are examined. SWCNTs and MWCNTs are used in this model. With the help of

Table 1: The numerical values of skin fraction $\tilde{C}_{f}=\frac{\mu_{n f}}{\mu_{f}} f^{\prime \prime}(0)$, when $\sigma=0.04$.

\begin{tabular}{|l|l|l|l|l|}
\hline $\boldsymbol{A}_{1}$ & $\mathbf{A}_{2}$ & $\mathbf{M}$ & $\boldsymbol{Q}$ & $\tilde{C}_{f}$ \\
\hline 0.1 & 0.2 & 0.3 & 0.5 & 0.439391 \\
\hline 0.3 & & & & 0.438104 \\
\hline 0.5 & 0.2 & & & 0.438885 \\
\hline & 0.4 & & & 0.438213 \\
\hline & 0.6 & 0.3 & & 0.437078 \\
\hline & & 0.7 & & 0.478820 \\
\hline & & 1.0 & & 0.453513 \\
\hline & & 1.3 & -1.5 & 0.437078 \\
\hline & & & -0.1 & -3.831310 \\
\hline & & & 0.1 & -1.162610 \\
\hline
\end{tabular}

Table 2: The numerical values of Nusselt number $N u_{x}=-\frac{k_{n f}}{k_{f}}(1+R d) \theta^{\prime}(0)$, when $\sigma=0.04$.

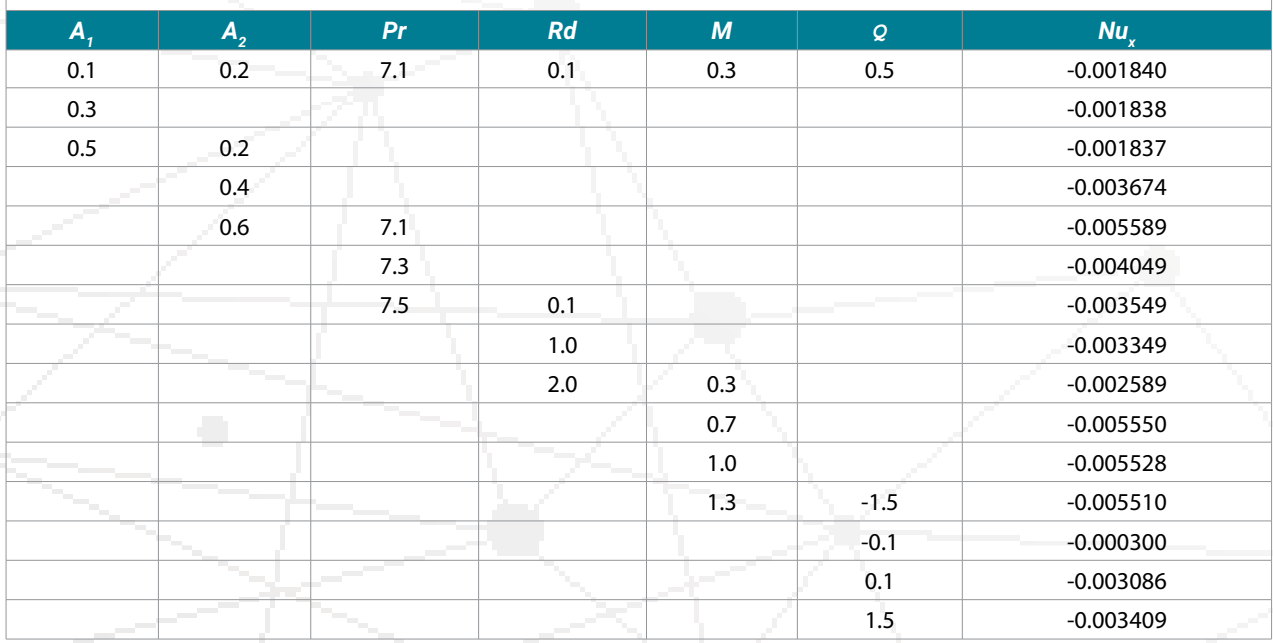




\begin{tabular}{|c|c|c|}
\hline Materials & SWCNT & MWCNT \\
\hline $\begin{array}{l}\text { Thermal Conductivity } \mathrm{k}_{\mathrm{nf}} \\
\mathrm{W} / \mathrm{mK}\end{array}$ & 3000 & 3000 \\
\hline $\begin{array}{l}\text { Electrical Conductivity } \otimes_{\mathrm{nf}} \\
\qquad \mathrm{S} / \mathrm{m}\end{array}$ & $10^{6} \cdot 10^{7}$ & $10^{6} \cdot 10^{7}$ \\
\hline Tensile Strength (GPa) & 150 & 150 \\
\hline Young's Modulus (GPa) & 1054 & 1200 \\
\hline
\end{tabular}

Table 4: The thermo physical properties of CNTs and nanofluids of some base fluids.

\begin{tabular}{|c|c|c|c|c|}
\multicolumn{2}{|c|}{ Physical Properties } & Specific Heat $\boldsymbol{c}_{p}\left(\mathbf{k g}^{-1} / \mathbf{k}^{-1}\right)$ & $\left(\mathbf{k g} / \mathbf{m}^{3}\right)$ Density & Thermal Conductivity $\mathbf{k}(\mathbf{W} / \mathbf{m k})$ \\
\hline \multirow{3}{*}{ Base fluid } & Water & $4.197 \times 10^{3}$ & $9.97 \times 10^{2}$ & $6.13 \times 10^{-1}$ \\
\cline { 2 - 5 } & Kerosene (lamp) oil & $2.090 \times 10^{3}$ & $7.83 \times 10^{2}$ & $1.45 \times 10^{-1}$ \\
\cline { 2 - 5 } & Engine oil & $1.910 \times 10^{3}$ & $8.84 \times 10^{2}$ & $1.44 \times 10^{-1}$ \\
\hline \multirow{2}{*}{ Nano fluids } & SWCNT & $4.25 \times 10^{2}$ & $2.6 \times 10^{3}$ & $6.6 \times 10^{3}$ \\
\cline { 2 - 5 } & MWCNT & $7.96 \times 10^{2}$ & $1.6 \times 10^{3}$ & $3 \times 10^{3}$ \\
\hline
\end{tabular}

Table 5: Thermal conductivity $\left(\mathrm{k}_{\mathrm{nf}}\right)$ of CNTs with different volume fraction $(\varphi)$.

\begin{tabular}{|c|c|c|}
\hline$\varphi$ & $\boldsymbol{k}_{n f}$ for SWCNT & $\boldsymbol{k}_{n f}$ for MWCNT \\
\hline 0 & $1.45 \times 10^{-1}$ & $1.45 \times 10^{-1}$ \\
\hline 0.01 & $1.74 \times 10^{-1}$ & $1.72 \times 10^{-1}$ \\
\hline 0.02 & $2.04 \times 10^{-1}$ & $2 \times 10^{-1}$ \\
\hline 0.03 & $2.35 \times 10^{-1}$ & $2.28 \times 10^{-1}$ \\
\hline 0.04 & $2.66 \times 10^{-1}$ & $2.57 \times 10^{-1}$ \\
\hline
\end{tabular}

similarity variables, the system of governing partial differential equations is changed into ordinary differential equations. The impacts of embedded of parameters are shown graphically. The impacts of skin friction coefficient and local Nusselt number are shown through Table 1 and 2. On the achieved study, the key remarks are listed below.

1. The velocity function $f^{\prime}(\Gamma)$ escalates with the escalation in $(\bigotimes),(\beta)$ and suction parameter (i.e. $Q>0$ ), and reduces with the escalation in $A_{1},(M)$ and injection parameter (i.e. $Q>0$ ).

2. The velocity function $f^{\prime}(\Gamma)$ shows reducing behavior with escalating values of $\left(A_{2}\right)$ within the region $(0 \leq \Gamma \leq 0.5)$ and shows escalation behavior with escalating values of $\left(A_{2}\right)$ within the region $(0.5<\Gamma \leq 1)$.

3. The velocity function $g(\Gamma)$ shows escalation behavior with escalation in $(\phi)$ and show reducing behavior with escalation in $\left(A_{1}\right), A_{2}$ and $(M)$.

4. The temperature function $\theta(\Gamma)$ show escalating behavior with the escalation in $(\bigotimes)$ and $(R d)$ shows reducing behavior with the escalation in $\left(A_{1}\right)$ and $(\operatorname{Pr})$.

\section{References}

1. Haq RU, Nadeem S, Khan ZH, Noor NFM. Convective heat transfer in MHD slips flow over a stretching surface in the presence of carbon nanotubes. Phys B Condens Matter. 2015; 457: 40-47. Ref.: https://tinyurl.com/ybhuvpbe

2. Liu MS, Lin MCC, HI Te, CC Wang. Enhancement of thermal conductivity with carbon nanotube for nanofluids. Int Comm in Heat and Mass Transfer. 2015; 32: 1202-1210. Ref.: https://tinyurl.com/ycqbu7c9

3. Nadeem S, Lee C. Boundary layer flow of nanofluid over an exponentially stretching surface. Nanoscale Res Lett. 2012; 7: 94. Ref.: https://tinyurl.com/ybp2rygp

4. Ellahi R, Raza M, Vafai K. Series solutions of non-Newtonian nanofluids with Reynolds' model and Vogel's model by means of the homotopy analysis method. Math Comput Model. 2012; 55: 18761891. Ref.: https://tinyurl.com/y9tn4s3b

5. Nadeem S, Haq RU, Khan ZH. Numerical study of MHD boundary layer flow of a Maxwell fluid past a stretching sheet in the presence of nanopar-ticles. J Taiwanlnst Chem Eng. 2014; 45: 121-126. Ref.: https://tinyurl.com/y9hsb38v

6. Nadeem S, Haq RU. MHD boundary layer flow of a nano fluid past a porous shrinking sheet with thermal radiation. J Aerospace Eng. 2015; 28: 1943-5525. Ref.: https://tinyurl.com/y97mpwdv 
7. Nadeem S, Haq RU. Effect of thermal radiation for megnetohydrody- namic boundary layer flow of a nanofluid past a stretching sheet with convective boundary conditions. J Comput Theor Nanosci. 2013; 11: 32-40. Ref.: https://tinyurl.com/y96y4ddt

8. Choi SUS. Enhancing thermal conductivity of fluids with nanoparticles. Int mech eng congress and exposition. 1995; 66: 99-105. Ref.: https://tinyurl.com/ycq6by6d

9. Buongiorno J. Convective transport in nanofluids. J Heat Transf. 2006; 128: 240-250. Ref.: https://tinyurl.com/yamfme6z

10. Sheikholeslami M. Influence of magnetic field on nanofluid free convection in an open porous cavity by means of Lattice Boltzmann method. J of Molecular Liquids. 2017; 234: 364. Ref.: https://tinyurl.com/y9yxzq6f

11. Sheikholeslami M. Magnetic field influence on nanofluid thermal radiation in a cavity with tilted elliptic inner cylinder. J of Molecular Liquids. 2017; 229: 137-147. Ref.: https://tinyurl.com/y7jrhyy3

12. Sheikholeslami M. Magnetohydrodynamic nanofluid forced convection in a porous lid driven cubic cavity using Lattice Boltzmann method. J of Molecular Liquids. 2017; 231: 555-565. Ref.: https://tinyurl.com/y86kyh8f

13. Sheikholeslami M. Numerical simulation of magnetic nanofluid natural convection in porous media. Physics Letters A. 2017; 381: 494-503. Ref.: https://tinyurl.com/ybllfpc2

14. Sheikholeslami M. Numerical study of heat transfer enhancement in a pipe filled with porous media by axisymmetric TLB model based on GPU. Eur Phys J Plus. 2014; 129: 248.

15. Sheikholeslami M. CVFEM for magnetic nanofluid convective heat transfer in a porous curved enclosure. Eur Phys J Plus. 2016; 131: 413. Ref.: https://tinyurl.com/y99bt4sh

16. Sheikholeslami M, Rokni HB. Simulation of nanofluid heat transfer in presence of magnetic field: A review. Int J of Heat and Mass Transfer. 2017; 115: 1203-1233. Ref.: https://tinyurl.com/ybahhr9g

17. Sheikholeslami M, Rokni HB. Free convection of $\mathrm{CuOeH} 2 \mathrm{O}$ nanofluid in a curved porous enclosure using mesoscopic approach. Int J of Heat and Mass Transfer. 2017; 42: 15393. Ref.: https://tinyurl.com/y8jetqnk

18. Sheikholeslami M. Houman BR. Numerical simulation for impact of Coulomb force on nanofluid heat transfer in a porous enclosure in presence of thermal radiation. Int $J$ of Heat and Mass Transfer. 2018; 118: 823-831. Ref.: https://tinyurl.com/y99vplln

19. Sheikholeslami M, Ganji DD, Javed MY, Ellahi R. Effect of thermal radiation on magnetohydrodynamics nanofluid flow and heat transfer by means of two phase model. J Magnetism and Magnetic Materials. 2015; 374: 36-43. Ref.: https://tinyurl.com/y8kd9kv6

20. Elias MM, Miqdad M, Mahbubul IM, Saidur R, Kamalisarvestani $M$, et al. Effect of nanoparticle shape on the heat transfer and thermodynamic performance of a shell and tube heat exchanger. Int Commun Heat Mass Transfer. 2013; 44: 93-99. Ref.: https://tinyurl.com/y9owqk5n

21. Alfven H. Existence of electromagnetic-hydrodynamic waves. Nature. 1942; 150: 405-406. Ref.: https://tinyurl.com/y9x9jb2p

22. Shah Z, Gul T, Khan AM, Ali I, Islam S. Effects of hall current on steady three dimensional nonnewtonian nanofluid in a rotating frame with brownian motion and thermophoresis effects. J Eng Technol. 2017; 6: 280-296. Ref.: https://tinyurl.com/yb28z25t

23. Shah Z, Islam S, Gul T, Bonyah E, Khan MA. The electrical MHD and hall current impact on micropolar nanofluid flow between rotating parallel plates. Results Phys. 2018: 9: 1201-1214. Ref.: https://tinyurl.com/ybelycjt

24. Shah Z, Islam S, Gul T, Bonyah E, Khan MA. Three dimensional third grade nanofluid flow in a rotating system between parallel plates with Brownian motion and thermophoresis effects. Results Phys. 2018; 10: 36-45. Ref.: https://tinyurl.com/yc24emj2

25. Casson NA. A flow equation for pigment oil suspension of printing ink type. In: Mill CC (ed) Rheology of dispersed system. Pergamon Press. Oxford. 1959.

26. Mehmood Z, Mehmood R, Iqbal Z. Numerical Investigation of Micropolar Casson Fluid over a Stretching Sheet with Internal Heating. Commun Theor Phys. 2017; 67: 443-448. Ref.: https://tinyurl.com/y7xbr4rv

27. Iqbal Z, Mehmood R, Azhar E, Mehmood Z. Impact of inclined magnetic field on micropolar Casson fluid using Keller box algorithm. Eur Phys J Plus. 2017; 132: 175. Ref.: https://tinyurl.com/yarhf5cl

28. Megahe AM. Effect of slip velocity on Casson thin film flow and heat transfer due to unsteady stretching sheet in presence of variable heat flux and viscous dissipation. Open Journal of Fluid Dynamic. 2016; 6: 303-320. Ref.: https://tinyurl.com/y85wxkph 
29. Abolbashari MH, Freidoonimehr N, Rashidi MM. Analytical modeling of entropy generation for Casson nano-fluid flow induced by a stretching surface. Advanced Powder Technology. 2016; 2: 542-552. Ref.: https://tinyurl.com/y7fxzbae

30. Attia HA, Ahmed MES. Transient MHD couette flow of a Casson fluid between parallel plates with heat transfer. Ital J Pure Appl Math. 2010; 27: 19-38. Ref.: https://tinyurl.com/y8zbgv6h

31. Mukhopadhyay S. Casson fluid flow and heat transfer over a nonlinearly stretching surface. Chin Phys B. 2013; 27: 074701-074705. Ref.: https://tinyurl.com/yc6purfo

32. Qasim M, Noreen S. Heat transfer in the boundary layer flow of a Casson fluid over a permeable shrinking sheet with viscous dissipation. Eur Phys J Plus. 2014; 7: 129-137. Ref.: https://tinyurl.com/y9gm47zv

33. Maxwell JC. Electricity and magnetism. $3^{\text {rd }}$ edn. Clarendon, Oxford. 1904.

34. Casson NA. Flow Equation for Pigment Oil Suspensions of the Printing Ink Type, Rheology of Disperse Systems. Pergamon Press: New York, USA. 1959; 84-104. Ref.: https://tinyurl.com/ycb3o3z4

35. Hussanan A, Salleh MZ, Tahar RM, Khan I. Unsteady boundary layer flow and heat transfer of a Casson fluid past an oscillating vertical plate with Newtonian heating. PLoS ONE. 2014; 9: e108763. Ref.: https://tinyurl.com/yaep7gfk

36. Walicka A, Falicki J. Reynolds number effects in the flow of an electro rheological fluid of Casson type between fixed surface of revolution. Appl Math Comput. 2015; 250: 639-649. Ref.: https://tinyurl.com/y8yqa48z

37. Hussain ST, Rizwan-ul-Haq, Khan ZH, Nadeem S. Water driven flow of carbon nanotubes in a rotating channel. Journal of Molecular Liquids. 2016; 214: 136-144. Ref.: https://tinyurl.com/ydbw4xpm

38. Sheikholeslami M, Hatami D, Ganji DD. Nanofluid flow and heat transfer in a rotating system in the presence of a magnetic field. Journal of Molecular Liquids. 2014; 190: 112-120. Ref.: https://tinyurl.com/y8r7d73a

39. Ishaq M, Ali G, Shah Z, Islam S, Muhammad S. Entropy Generation on Nanofluid Thin Film Flow of Eyring-Powell Fluid with Thermal Radiation and MHD Effect on an Unsteady Porous Stretching Sheet. Entropy. 2018; 20: 412. Ref.: https://tinyurl.com/yb39tfy7

40. Nasir S, Islam S, Gul T, Shah Z, Khan MA, et al. Three-dimensional rotating flow of MHD single wall carbon nanotubes over a stretching sheet in presence of thermal radiation. Applied Nano science. 2018; 8: 1361-1378. Ref.: https://tinyurl.com/y9bpe8v9

41. Hammed H, Haneef $M$, Shah Z, Islam S, Khan W, et al. The Combined Magneto hydrodynamic and electric field effect on an unsteady Maxwell nanofluid Flow over a Stretching Surface under the Influence of Variable Heat and Thermal Radiation. Appl Sci. 2018; 8: 160. Ref.: https://tinyurl.com/ycfnfqpn

42. Ali A, Sulaiman M, Islam S, Shah Z, Bonyah E. Three-dimensional magnetohydrodynamic (MHD) flow of Maxwell nanofluid containing gyrotactic micro-organisms with heat source/sink. AIP Advances. 2018; 8: 085303. Ref.: https://tinyurl.com/yatjo8op 